\title{
Effectiveness of reinforcing kidney and activating blood therapy for premature ovarian failure: a systematic review and meta-analysis.
}

\author{
Kehuan Sun ${ }^{1,2 \#}$, Weihao Chen ${ }^{1 \#}$, Fengrong Wang ${ }^{3 \#}$, Chunming Yang ${ }^{4}$, Yurong Wang ${ }^{*}$, Ronghua \\ Zhang $^{*}$
}

${ }^{1}$ College of Traditional Chinese Medicine, Jinan University, 601 Huangpu West Avenue, Guangzhou, Guangdong, China

${ }^{2}$ Shenzhen Institute of Geriatric Medicine, Shenzhen, Guangdong Province-518000, China

${ }^{3}$ The Medical Center of Stomatology, the First Affiliated Hospital of Jinan University, Guangzhou, Guangdong, China

${ }^{4}$ Health Checkup Center, the First Hospital Affiliated To Army Medical University, 30 Gaotanyanzheng Street, Shapingba District, Chongqing, China

${ }^{\#}$ These authors contributed equally to this work.

\begin{abstract}
Purpose: This systematic review aimed to evaluate the effects of reinforcing kidney and activating blood (RKAB) therapy for patients with premature ovarian failure (POF) from randomized controlled trials (RCTs).

Methods: We searched 7 databases and screened 11 studies from 267 articles by excluded the studies which did not reach inclusion criteria. Studies selection and data extraction were performed by two reviewers independently. Finally, the Review Manager Software was utilized to pool data, and evaluated the overall level of the evidence using Grading of Recommendations Assessment, Development, and Evaluation (GRADE) criteria.

Results: RKAB therapy significantly improve the serum levels of follicle-stimulating hormone (FSH) (MD-5.50, 95\% CI: (-8.14, -2.86), P<0.0001), luteinizing hormone (LH) (MD-3.33, 95\% CI: (-5.09, -1.57), $P=0.0002)$ and estradiol (E2) (MD 6.82, 95\% CI: $(1.16,12.47), P=0.02)$ as well as clinical curative efficiency (OR 2.60, 95\% CI: $(1.73,3.89), P<0.00001)$ when compared with hormone replacement therapy (HRT) group.

Conclusion: The current evidence on RKAB therapy for POF is insufficient to draw a firm conclusion due to scarcity of studies with a low risk of bias and likely publication bias. Future rigorously designed studies are needed to confirm the effectiveness of RKAB therapy in patients with POF.
\end{abstract}

Keywords: Premature ovarian failure, Reinforcing kidney, Activating blood therapy, Meta-analysis.

\section{List of Abbreviations}

RKAB: Reinforcing Kidney and Activating Blood; POF: Premature Ovarian Failure; RCT: Randomized Controlled Trials; FSH: Follicle-Stimulating Hormone; LH: Luteinizing Hormone; E2: Estradiol; HRT: Hormone Replacement Therapy; AEs: Adverse Effect; TCM: Traditional Chinese
Medicine; OR: Odds Ratios; CI: Confidence Intervals; MD: Mean Differences; GRADE: The Grading of Recommendations Assessment, Development and Evaluation; HPOA: Hypothalamic-Pituitary-Ovary Axis; INH-b: Serum Inhibin B.

\section{Introduction}

Premature ovarian failure (POF) is a serious disease that affects women's reproductive, physical and mental health. Often accompanied by symptoms of perimenopause, such as hot flushes, vaginal dryness, dysphoric and night sweats, which are similar to those of a natural menopause [1]. The morbidity
Accepted on December 28, 2018

of POF is approximately $1 \%-3 \%$, accounted for secondly amenorrhea $2 \%-10 \%$, and there is on the rise in recent years.

Although some researches of POF are given to explain, most of the reasons were gene mutations, autoimmune disorders and environmental factors, the causes of POF remain unclear so far [2]. Most experts recommend that estrogen and progestin replacement therapy is an appropriate option for POF patient. 
Although hormone replacement therapy (HRT) as a regular treatment were recommend by most experts [3], it has an inevitable adverse effect (AEs), like increase risks of strokes, breast cancer, heart attacks, and endometrial cancer [4]. Therefore, it needs to consider whether there is a better alternative therapy to displace HRT in women with POF.

Premature ovarian failure was defined as "Xueku" or "Xuejie" in Traditional Chinese Medicine (TCM) [5,6]. Chinese Herbal already has been used to alleviate the symptoms of POF in the past $2000 \mathrm{y}$, especially reinforcing kidney and activating blood (RKAB) therapy [7,8]. It is a kind of decoctions which belong to TCM and compose of Chinese herbal medicine such as "Shengdi" "Shudi" "Tusizi" "Yinyanghuo" [9]. Moreover, it has been found to be effective in relieving POF symptoms [10]. However, there is no systematic review of the efficacy effects of RKAB on POF. Therefore, it is necessary to summarize and evaluate the current evidence from RCTs on the effects of RKAB in patients with POF.

\section{Materials and Methods}

\section{Criteria for considering studies for this review}

Types of studies: Included all RCTs of RKAB in the treatment of POF. No randomized trials, animal work, case reports or series, theory research, review article, letters, qualitative studies were excluded.

Types of participants: Women who were diagnosed with POF were considered. The diagnostic criteria for POF: 1) Women who were less than 40 y old; 2) Twice serum FSH levels detection above $40 \mathrm{IU} / \mathrm{L}$ (interval time of detection at least 1 month or more); 3) Amenorrhea for 4 months or more. Other diseases such as insensitive ovarian syndrome, polycystic ovary syndrome and diminished ovarian reserve were excluded.

Types of interventions: We included trials in which decoction of RKAB therapy, regardless of formulas of decoction. Other therapy like strengthening spleen or smoothing liver as an additional were also included as long as RKAB is the primary intervention method. We excluded the studies which combined RKAB with other TCM therapy such as acupuncture or electro-acupuncture.

Types of control groups: We included the trials which adopted the HRT conventional treatments for a control group. Moreover, the trials of both treatment and control group adopted HRT therapy were also included. The treatment comparisons investigated in this review listed below.

\section{RKAB alone versus HRT}

\section{RKAB plus HRT versus HRT}

Types of outcome measures: Serum FSH levels and clinical curative efficiency were primary outcomes. Serum luteinizing hormone (LH) and estradiol (E2) levels were secondary outcomes.

\section{Search strategy}

We have searched three International and four Chinese databases, the following electronic databases:

1. Medline (1946 to October 14, 2017);

2. EMBASE (1974 to October 14, 2017);

3. Cochrane Central Register of Controlled Trials (CENTRAL);

4. China National Knowledge Infrastructure (CNKI, 1979 to October 14, 2017);

5. Wanfang DATA (1980 to October 14, 2017);

6. VIP (Wei Pu Information, 1989 to October 14, 2017);

7. China Biological Medicine Database (1978 to October 14, 2017).

Various combinations of Mesh headings and keywords were used, including "premature ovarian failure", "premature ovarian insufficiency", "bushenhuoxue", "bushen huoxue" et al. All the publication languages were restricted to Chinese and English.

\section{Study selection}

Two reviewers (Weihao and Yuron) examined titles and abstracts independently retrieved from the search results to select the potential studies. Then checked the studies whether meet the inclusion criteria by the same reviewer (Weihao and Yurong) independently. The studies which did not meet the inclusion criteria were excluded. And the authors' name, title of article and institution were blinded to reviewer for avoiding subjectivity. We discussed disagreement with the third author (Min) to reach consensus.

\section{Data extraction}

Two reviewers (Weihao and Guijuan) extracted data independently. And we resolved all the disagreement by discussed with another reviewer (Min). The information (design type, randomization and blinding method, inclusion/ exclusion criteria and number of patients, detail of RKAB intervention, comparison group, FSH, LH and E2 level, clinical curative efficiency, withdrawal, adverse events (AEs), and follow-up time were extracted by the same reviewers (Weihao and Guijuan).

\section{Risk of bias assessment}

Two reviewers (Weihao and Xiaoqian) evaluated the risk of bias of the included studies which provided by the Cochrane Collaboration [11]. And we examined the risk of bias based on seven parts, which of selection bias (random sequence generation and allocation concealment), performance bias (blinding of participants and personnel), detection bias (blinding of outcome assessment), attrition bias (incomplete outcome data), reporting bias (selective reporting), and other bias. We resolved all the disagreements between two authors 
by discussing with the third author (Min Ma) to reach consensus.

\section{Assessment of heterogeneity}

We evaluated clinical heterogeneity by calculating the $\mathrm{I}^{2}$ statistic which indicates the weight of variability among studies rather than sampling error. If heterogeneity more than $50 \%\left(\mathrm{I}^{2}\right.$ $50 \%$ ), we would synthesize the data with the random-effect model, if no significant statistical heterogeneity or heterogeneity less than $50 \%\left(\mathrm{I}^{2} 50 \%\right)$, we would use the fixedeffect model to synthesize the data.

\section{Assessment of reporting biases}

We intended to draw funnel plots to evaluate the reporting biases when more than 10 trials usually. Funnel plots suggested the potential reporting biases by visual asymmetry which could cause by publication bias or other reasons.

\section{Data synthesis}

The Review Manager (RevMan) Software (Version 5.3 Copenhagen: The Nordic Cochrane Centre, The Cochrane Collaboration, 2014) was utilized to pool and analyse data. We divided the trials into subgroup based on the type of intervention (RKAB alone and RKAB as an adjunct), type of outcome measure (hormone levels and clinical curative efficiency). Since the clinical curative efficiency is the dichotomous variable, we pooled the data and expressed as odds ratios (OR) and 95\% confidence intervals (CI) to describe the results. For the hormonal outcomes, it belongs to continuous variables; therefore the data were pooled and expressed as mean differences (MD) and 95\% confidence intervals $(\mathrm{CI})$.

\section{Quality of evidence}

We used "the grading of recommendations assessment, development and evaluation, (GRADE)" which is a method of grading quality of evidence and strength of recommendations and were created by the GRADE Working Group to evaluate the level of evidence and summarize outcome. The GRADEpro software (version 3.6.1, Grade Working Group) was used.

\section{Results}

\section{Results of the search}

The original search results screened and identified 267 articles. 241 studies were excluded according to the title and abstract and retrieved 26 studies for more detailed assessment. Then we exclude 15 studies based on additional reports of include trials not contributing data, unreasonable study design and included 11 studies which met the inclusion criteria in this systematic review finally (Figure 1).

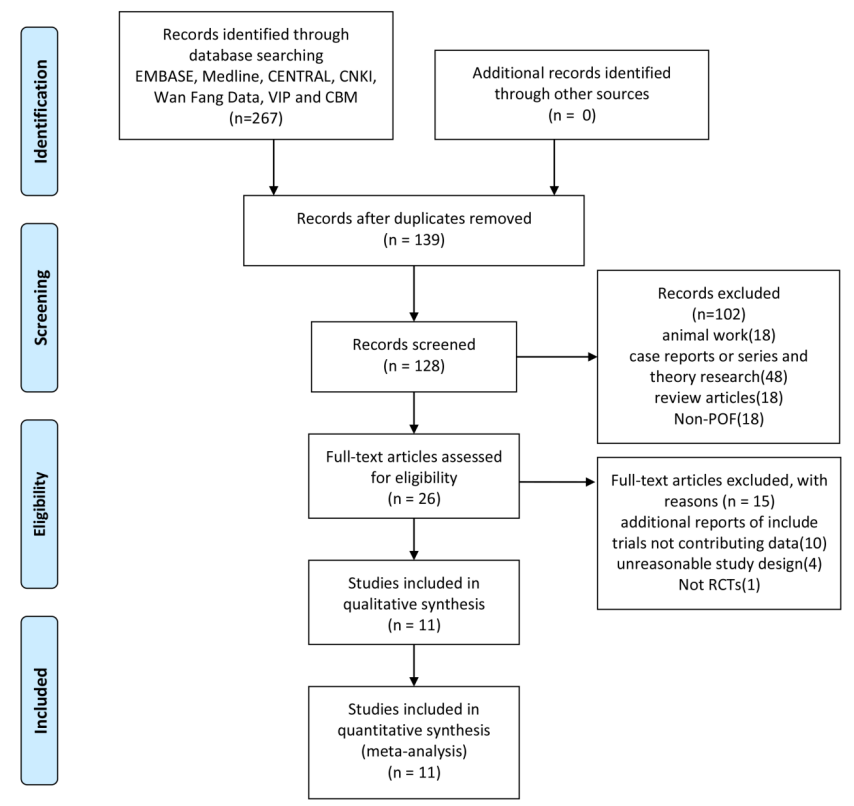

Figure 1. PRISMA flow diagram.

\section{Included studies}

Eleven RCTs studies, including 1044 participants (534 in experiment group and 510 in control group) reached the inclusion criteria. All studies originated from China and published in Chinese medical literature databases and went through the peer-reviewed process.

\section{Participants}

The patients in seven trials [12-18] were recruited from outpatient, a part of patients were recruited from both outpatient and inpatient in one trial [19], and the patients in rest trials [20-22] were not specifically described. Four $[12,13,15,16]$ and Six studies [12-16,22] did not clearly report the patients' average age and duration of amenorrhea respectively.

\section{Interventions}

RKAB therapy was carried out alone in six trails [12-14,17-19] in the treatment group. Another five trails [15,16,20-22] $\mathrm{RKAB}$ as an adjunct intervention. The treatment length ranged from 1 to 6 months (Table 1).

Table 1. The characteristics of included studies.

\begin{tabular}{lllllll}
\hline Author & $\begin{array}{l}\text { Sample } \\
(\mathrm{E} / \mathrm{C})\end{array}$ & size & Mean age (E/C) & Intervention & Comparison & $\begin{array}{l}\text { Outcome } \\
\text { measures }\end{array}$
\end{tabular}




\begin{tabular}{|c|c|c|c|c|c|c|c|}
\hline Ding [12] & $60 / 60$ & NR & RKAB & HRT & FSH, LH, E2, CCE & $\begin{array}{l}\text { After treatment ( } 5 \\
\text { months) }\end{array}$ & NR \\
\hline Dong [13] & $30 / 30$ & $N R$ & RKAB & HRT & FSH, LH, E2, CCE & $\begin{array}{l}\text { After treatment ( } 3 \\
\text { months) }\end{array}$ & NR \\
\hline Hong [20] & $31 / 31$ & $34.2 \pm 0.5 / 33.8 \pm 0.5$ & RKAB and HRT & HRT & FSH, LH, E2, CCE & $\begin{array}{l}\text { After treatment ( } 3 \\
\text { months) }\end{array}$ & 12 months \\
\hline Hou [14] & $100 / 100$ & $34.57 \pm 4.33 / 33.87 \pm 4.49$ & RKAB & HRT & FSH, LH, E2 & $\begin{array}{l}\text { After treatment ( } 3 \\
\text { months) }\end{array}$ & NR \\
\hline Lu [15] & $30 / 30$ & $N R$ & RKAB and HRT & HRT & FSH, LH, E2, CCE & $\begin{array}{l}\text { After treatment }(3 \\
\text { months and } 6 \\
\text { months) }\end{array}$ & NR \\
\hline Ruan [16] & $20 / 20$ & NR & RKAB and HRT & HRT & FSH, LH, E2, CCE & $\begin{array}{l}\text { After treatment }(3 \\
\text { months and } 6 \\
\text { months) }\end{array}$ & NR \\
\hline Shi [21] & $80 / 80$ & $34.9 \pm 2.1 / 35.2 \pm 2.3$ & RKAB and HRT & HRT & $\mathrm{FSH}, \mathrm{LH}, \mathrm{E} 2$ & $\begin{array}{l}\text { After treatment } \\
(21 \text { days and } 6 \\
\text { months })\end{array}$ & NR \\
\hline Teng [17] & $42 / 21$ & $31 \pm 5 / 28 \pm 5$ & RKAB & HRT & $\mathrm{FSH}, \mathrm{LH}, \mathrm{E} 2, \mathrm{CCE}$ & $\begin{array}{l}\text { After treatment ( } 6 \\
\text { months) }\end{array}$ & NR \\
\hline Wang [18] & $20 / 20$ & $34.53 \pm 4.32 / 33.72 \pm 6.48$ & RKAB & HRT & FSH, LH, E2, CCE & $\begin{array}{l}\text { After treatment ( } 3 \\
\text { months) }\end{array}$ & NR \\
\hline Zheng [19] & $30 / 30$ & $36.43 \pm 4.17 / 35.78 \pm 4.81$ & RKAB & HRT & FSH, LH, E2, CCE & $\begin{array}{l}\text { After treatment (1 } \\
\text { month) }\end{array}$ & 6 months \\
\hline Zhou [22] & $91 / 88$ & $33 \pm 2.2$ & RKAB and HRT & HRT & FSH, LH, E2, CCE & $\begin{array}{l}\text { After treatment } \\
\text { (21 days) }\end{array}$ & NR \\
\hline
\end{tabular}

Note: E/C: Experimental group/Control group; NR: Not Reported; RKAB: Reinforcing Kidney and Activating Blood; HRT: Hormone Replacement Therapy; FSH: FollicleStimulating Hormone; LH: Luteinizing Hormone; E2: serum Estradiol; CCE: Clinical Curative Efficiency.

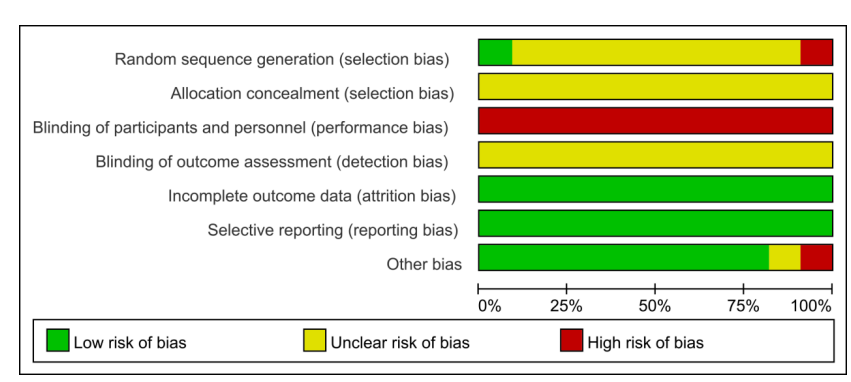

Figure 2. Risk of bias graph.

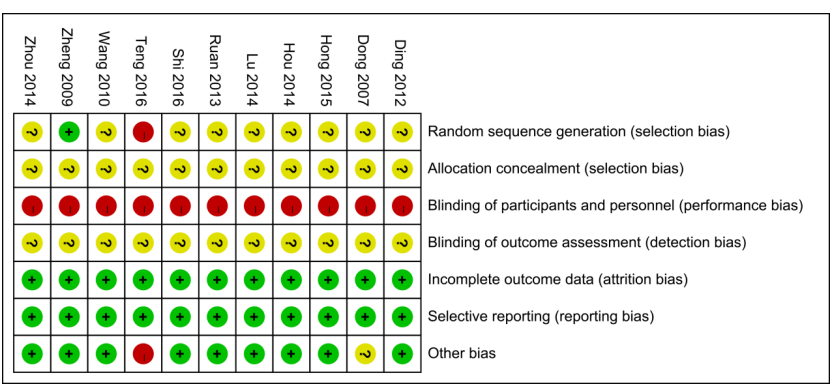

Figure 3. Risk of bias summary.

\section{Risk of bias in included studies}

Among the 11 included articles, 9 studies [12-16,18,20-22] did not describe randomization procedures inadequately, therefore we rated an unclear risk of bias, the others studies [17] described an inadequate random sequence generation by patient preference. None of the trials describe adequate allocation concealment specifically; we rated an unclear risk of bias to all studies. For participant and personnel assessment blinding, because none of the trials design placebo group, it very likely patients and personnel were not blind, therefore we assessed a high risk of bias to all studies. We evaluated an unclear risk of bias to all trails because we couldn't find any information about blinding of outcome assessment. None of the included studies had any losses, so we assessed a low risk of bias for incomplete outcome data to all trials. All of the included trials were at low risk of bias for selective reporting. One trial [17] was at high risk of bias for another source because the patient's average age of the treatment group was higher than the control group $(\mathrm{P}<0.05)$, the other trial [13] did not describe the statistical discrepancy of patient's average age between treatment group and control group, so we assessed an unclear risk of bias (Figures 2 and 3).

\section{Effects of RKAB}

Primary outcomes: FSH serum level: The level of serum FSH was reported by all studies. The test of heterogeneity was considerable heterogeneity $\left(\mathrm{I}^{2}=90 \%, \mathrm{P}<0.00001\right)$, therefore we pool the data with the random-effect model. The results indicated a statistically significant difference in FSH level between the RKAB group and the control group (MD -5.50, 95\% CI: $-8.14,-2.86, \mathrm{P}<0.0001)$ which suggests that RKAB 
therapy can significant decrease the serum FSH level in the treatment group than control group (Figure 4).

Clinical curative efficiency: Clinical curative efficiency was reported in nine studies [12,13,15-20,22]. The test of heterogeneity was low heterogeneity $\left(\mathrm{I}^{2}=2 \%, \mathrm{P}=0.42\right)$, therefore we pool the data with the fixed-effect model. The results indicated that statistically significant difference in the clinical curative efficiency in the RKAB group than the control group (OR 2.60, 95\% CI: 1.73, 3.89, $\mathrm{P}<0.00001$ ) which suggests that RKAB therapy can prominently improve the clinical curative efficiency in the treatment group when compared with the control group (Figure 5).

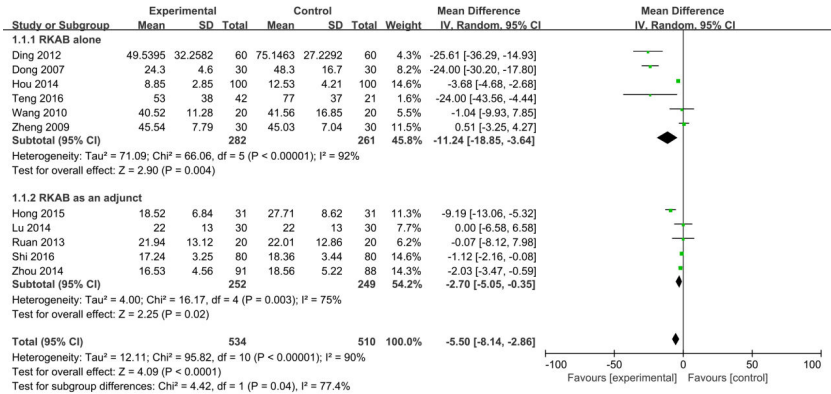

Figure 4. The serum level of follicle-stimulating hormone (FSH).

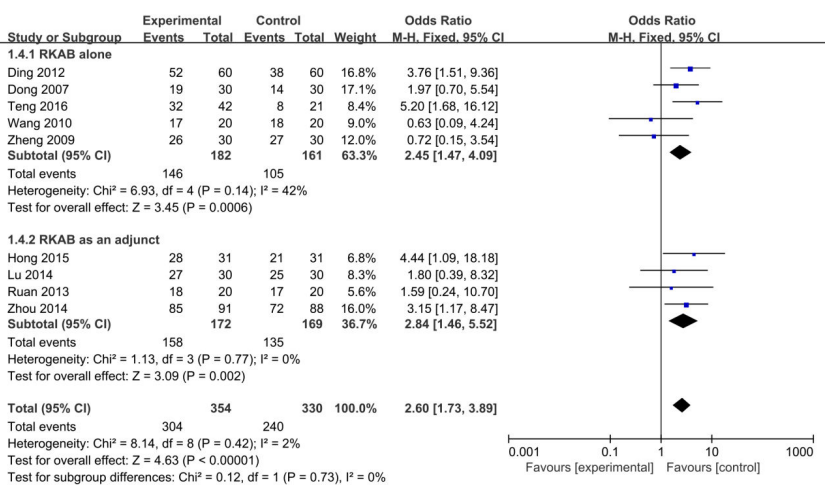

Figure 5. The impact of RKAB therapy on clinical curative efficiency.

Secondary outcomes: E2 serum level: The level of serum E2 was reported by all studies. The test of heterogeneity was considerable heterogeneity $\left(\mathrm{I}^{2}=97 \%, \mathrm{P}<0.00001\right)$, therefore we pool the data with the random-effect model. The results indicated a statistically significant difference between the RKAB and control group (MD 6.82, 95\% CI: 1.16, 12.47, $\mathrm{P}=0.02$ ) which suggests that $\mathrm{RKAB}$ therapy can prominently increase the serum E2 level in the treatment group than the control group. The studies of RKAB alone [12-14,17-19] found no statistically significant difference between the groups, while the studies of RKAB as an adjunct [15,16,20-22] found a statistically significant difference (Figure 6).

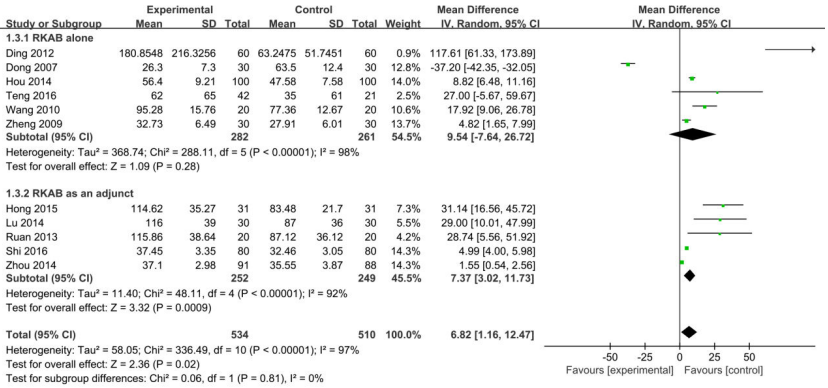

Figure 6. The serum level of estradiol (E2).

LH serum level: The level of serum LH was reported by all studies. The test of heterogeneity was considerable heterogeneity $\left(\mathrm{I}^{2}=89 \%, \mathrm{P}<0.00001\right)$, therefore we pool the data with the random-effect model. The results indicated a statistically significant difference in LH levels between the RKAB group and the control group (MD -3.33, 95\% CI: -5.09, -1.57, $\mathrm{P}=0.0002$ ) which suggests that RKAB therapy can prominently decrease the serum LH level in the treatment group than the control group (Figure 7).

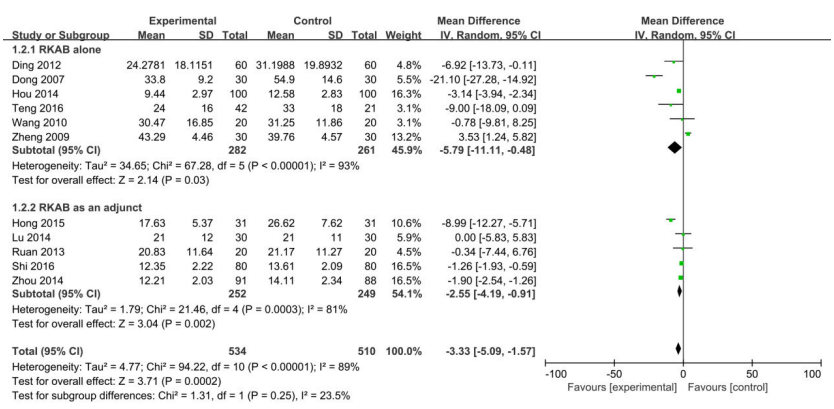

Figure 7. The serum level of luteinizing hormone (LH).

Adverse effects and other: Among the included studies, adverse effects of RKAB just reported in one trial [21]. Shi reported that there was no serious side effect. One study [13] reported ultrasound astral follicles counts. Two studies $[16,18]$ reported anti-ovary antibodies. Wang mentioned that they detected anti-zona pellucida antibodies and anti-ovary antibodies. Ruan reported that they only detected anti-ovary antibodies.

\section{Publication bias}

We included sufficient studies in this systematic review so we can make a funnel plot for publication bias of serum hormone level and clinical curative efficiency respectively. From the funnel plot, it suggested that there were potential publication biases by visual asymmetry in this meta-analysis (Figures 8 and 9). 


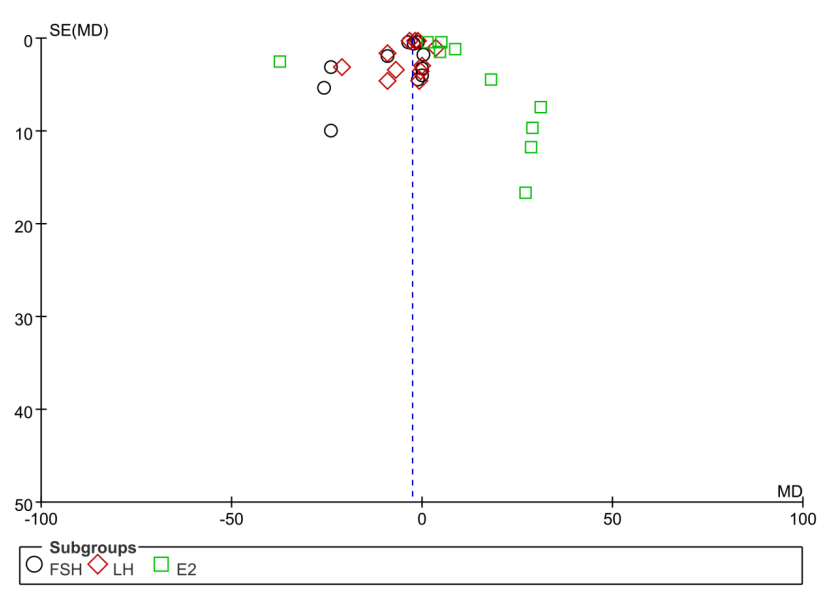

Figure 8. Funnel plot of serum hormones level.

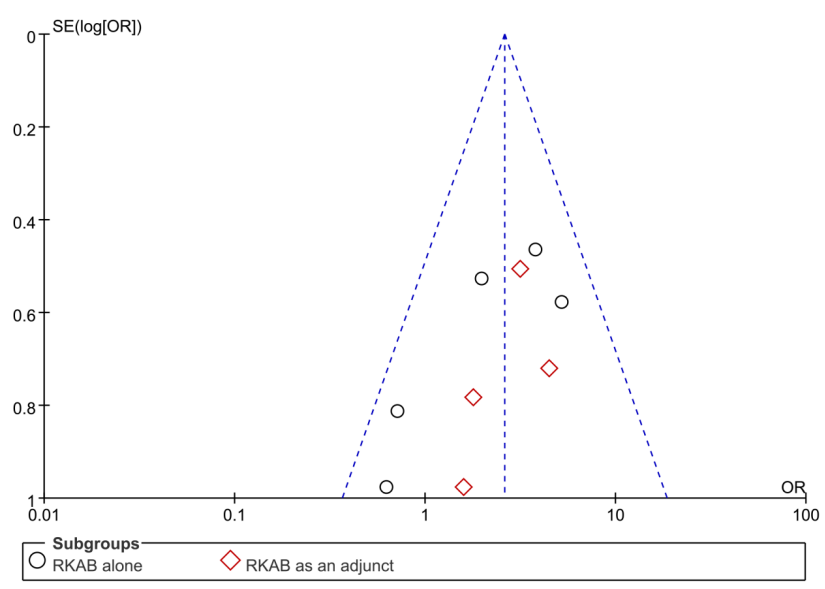

Figure 9. Funnel plot of clinical curative efficiency.

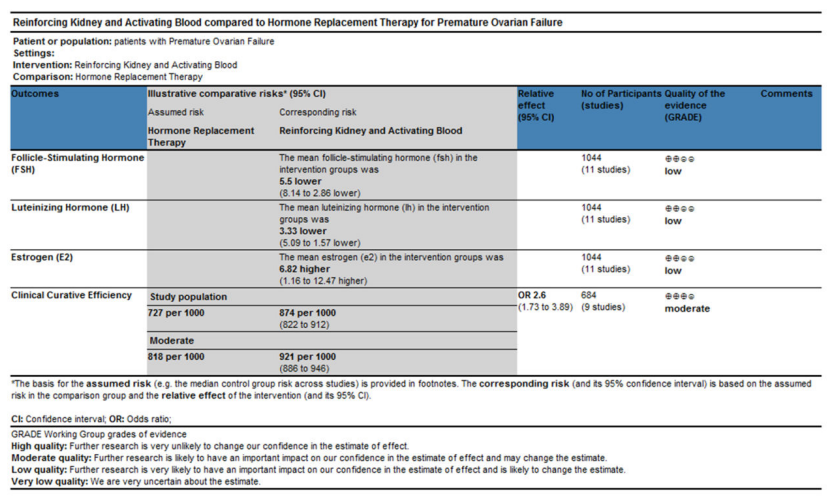

Figure 10. Level of evidence (GRADE).

\section{Sensitivity analysis}

We perform sensitivity analysis in this systematic review by change the mode of statistic (fixed-effect and random-effect model) and effect size in serum hormone levels (MD and SMD) and clinical curative efficiency (OR and RR). Even so, we found that the results were not changed.

\section{Quality of evidence}

The level of evidence was assessed according to GRADE from low to moderate (Figure 10). We assessed all outcomes a downgrade primally because blinding, randomization process and allocation concealment were not mentioned in most of the studies. Furthermore, we assessed an inconsistency of all outcomes downgraded except clinical curative efficiency.

\section{Discussion}

\section{Summary of main finding}

From the outcomes in this systematic review, it has indicated that RKAB therapy could significant decrease FSH and LH serum levels, increase E2 levels as well as improve the efficiency of clinical curative on POF patients. The results seem favorable in all outcomes however it should be interpreted carefully because of the high risk of bias of binding and reporting. We assessed a "moderate" level to clinical curative efficiency using GRADE. The quality of evidence of FSH, E2 and LH serum level were "low" because of the serious risk of bias and inconsistency.

\section{Symptoms score scale}

Four studies $[14,15,17,18]$ reported symptoms score scale in included studies. However, the symptoms scores scale was various. Wang et al. did not mention the detailed name of symptoms score scale; Hou reported the modified Kupperman scale, while Teng reported TCM symptoms score scale. Therefore, these data couldn't be combined in this metaanalysis.

\section{Potential mechanism of RKAB in POF}

As a result of modern medical research, the cause of premature ovarian failure was various and complicated which include chromosome aberrations, gene mutations, autoimmunity, congenital enzyme reduction or lack of, the barrier of gonadotropin, infection factors, reproduction factors, social psychological factors or environment pollution [23]. RKAB therapy has an effect in POF possibility by regulating hypothalamic-pituitary-ovary axis (HPOA) [24]. Beyond that, RKAB therapy may regulate the autonomic nervous system, increase blood flow in ovarian, improve the level of INH-b (serum inhibin B) and improve the reactive of ovarian response to FSH to have an effect on POF [25]. From the etiology and pathogenesis in TCM, "Xueku" which is the disease as same as POF was caused by kidney-deficiency, spleen-deficiency, blood-deficiency, qi stagnation and blood stasis or phlegm stagnation [26]. Like HPOA, there also was a similar theory in TCM which of kidney-tiangui-chongren-uterus axis [27]. Dominated by the kidney, regulate by Tiangui, through the full and empty of blood in chongren, finally have an effect on the ovary. Although there are various hypotheses, we couldn't know which one is closer to the truth. 


\section{Applicability of evidence}

In this review, we include eleven trials to assess RKAB therapy in the treatment of patients with premature ovarian failure. As a result, we founded that $\mathrm{RKAB}$ therapy significantly decreased FSH serum level and LH serum level, while increase E2 serum levels as well as improvement in clinical curative efficiency. The result seems favorable in all outcomes of pool data. However, it should be interpreted carefully due to the unclear risk of selection bias, the high risk of performance bias, the unclear risk of detection bias and publication bias. Furthermore, we couldn't make a confirm conclusion of which RKAB therapy could maintain the long-term efficacy because just two studies $[19,20]$ reported follow-up time. It needs more rigorous study design, large sample size and follow-up to ensure RKAB therapy actually could improve hormone levels and clinical curative efficiency no matter in short-term or longterm. In addition, without the adequate method of allocation concealment and blinding, the final results of the intervention effect may be exaggerated [28,29]. Although Chinese herbals of placebo were adopted in other trials to exclude placebo effect, none of included studies set a placebo group in this systematic review. However, placebo effects in biochemical parameters were less sensitive than some subjective symptoms such as gastric, hot flushes and dysphoric according to a recent study [30]. In this review, the serum hormone levels belong to biochemical parameters. So how much it will affect the outcomes of hormone levels without a placebo group, this is need to be considered.

\section{Conclusion}

This systematic review and meta-analysis suggest that RKAB therapy could improve the serum levels of the hormone as well as improvement in the clinical curative efficiency in patients with POF. However, because of the high risk of bias and insufficient follow-up studies, it is necessary to carry out more rigorously designed studies to confirm the effectiveness in patients with POF.

\section{Conflict of Interest}

The authors declare that they have no competing interests.

\section{Acknowledgement}

This work is supported by Natural Science Foundation of Guangdong Province (NO. 2018A0303130158), Shenzhen Science and Technology Program (No.JCYJ20160428110235852).

\section{References}

1. Chen W, Shi Y. General situation about etiology pathogenesis and clinical and experimental research of Traditional Chinese treatment of premature ovarian failure. J Liaoning Univ Trad Chinese Med 2014; 12: 205-208.
2. Chai S, Li S, Xia T. Discuss and prospect on etiology and pathogenesis of premature ovarian failure. Hubei J Trad Chinese Med 2010; 7: 76-79.

3. Tao M. Hormone replacement therapy in the treatment of premature ovarian failure. J Develop Med (Electronic Version) 2015; 2: 65-67.

4. Shelling AN. Premature ovarian failure. Reproduction 2010; 140: 633-641.

5. He A. TCM in the treatment of premature ovarian failure. Jilin J Trad Chinese Med 2007; 7: 1-2.

6. Yan D, Shi Y. Consideration of the recognition and current results of treatment of premature ovarian failure in TCM. Jilin J Trad Chinese Med 2010; 6: 548-550.

7. $\mathrm{Xu} \mathrm{X}$, Shi X. Review of reinforing kidney decoction in the treatment of gynecologic diseases. J Pract Trad Chinese Med 2012; 7: 615-616.

8. Gao H, Xia T, Han B, Yang J. Chinical study of Bushen Tiaochong recipe on treating premature ovarian failure. Liaoning J Trad Chinese Med 2007; 11: 1557-1560.

9. Ge S. Consideration of the recognition and prevention of premature ovarian failure in TCM. China J Chinese Med 2010; 2: 306-307.

10. Jin Z, Huang X, Yang Y, Wang L, He D, Liu W. Treatment of premature ovarian failure by Bushen Huoxue recipe combined estrogen and progesterone: a clinical research. Chinese J Integr Trad West Med 2013; 5: 586-589.

11. Higgins JPT, Green S. Cochrane Handbook for systematic reviews of interventions Version 5.0.1 2008.

12. Ding L. Clinical observation on Bushen Jianpi and Tiaogan Huoxue method in the treatment of premature ovarian failure. Guide China Med 2012; 10: 570-571.

13. Dong L, Jiang L, Lin X, Meng W, Zhu N. Effects of Bushen Huoxue decoction on serum FSH, LH, E2 and PRL in patients. Shanghai J Trad Chinese Med 2007; 11: 48-49.

14. Hou A, Wang L, Du J, Fang Y, Xu Y. Clinical research of Bushen Huoxue Decoction in the treatment of premature ovarian failure. Henan Trad Chinese Med 2014; 2: 314-316.

15. Lu D, Gao M. The curative effect of Chinese herbals combination with hormone therapy in the treatment of premature ovarian failure. Hebei Med J 2014; 36: 1210-1211.

16. Ruan R, Yi Y. Clinical observation on Chinese herbals combination with hormon therapy for treatment of premature ovarian failure in 20 patients. J Sichuan Trad Chinese Med 2013; 31: 94-95.

17. Teng $\mathrm{X}, \mathrm{Li} \mathrm{P}$. The curative effect of Jianpi Bushen Huoxue decoction in the treatment of premature ovarian failure with Spleen-Kidney Yang deficiency. Chinese J Integr Trad West Med 2016; 1: 119-122.

18. Wang H, Zhu X, Hu L, Liang R. Clinical study of Bushen Huoxue Method on premature ovarian failure with the immunologic unbalance. Jiangxi Med J 2010; 12: 1172-1174. 
19. Zheng C, Yu N, Wang Y. Clinical observation on Bushen Tiaogan Huoxue decotcion in the treatment of 30 patients with premature ovarian failure. Hunan J Trad Chinese Med 2009; 3: 40-42.

20. Hong L. Effect analysis of Chinese herbals combination with hormone therapy in the treatment of 31 patients with premature ovarian failure. Chinese Foreign Med Res 2015; 13: 33-34.

21. Shi Y. The curative effect of estrogen and progesterone combined with Bushen Huoxue decoction in the treatment of premature ovarian failure. Int Med Health Guide News 2016; 22: 651-653.

22. Zhou Y. Effect analysis of Bu Shen Huo Xue decoction combined with estrogen and progesterone hormones in the treatment of premature ovarian failure. Chinese Community Doctors 2014; 16: 91-92.

23. Zhao Q, Ye C, Li W. Research status and progress of premature ovarian failure. Reprod Contracept 2014; 1 : 59-64.

24. Zhao X, Cui F, Wang X. A review on mechanism of promotion ovulation in kidney medicine. Clin J Chinese Med 2011; 2: 1-3.

25. Jia N. Research on mechanism of reinforcing kidney and activating blood therapy in treatment of premature ovarian failure. J Pract Gynecol Endocrinol 2015; 2: 22.

26. Kang Y, Man Y, Leng Y, Zhao X. Premature ovarian failure study on the pathogenesis of TCM and Western medicine. Guangming J Chinese Med 2015; 2: 358-359.

27. Yang J. An important part of menstruation kidney-tianguichongren-uterus axis. J Sichuan Trad Chinese Med 1983; 2: $14-16$.

28. Pildal J, Hrobjartsson A, Jorgensen KJ, Hilden J, Altman DG, Gotzsche PC. Impact of allocation concealment on conclusions drawn from meta-analyses of randomized trials. Int J Epidemiol 2007; 36: 847-857.
29. Wood L, Egger M, Gluud LL, Schulz KF, Juni P, Altman DG. Empirical evidence of bias in treatment effect estimates in controlled trials with different interventions and outcomes: meta-epidemiological study. BMJ 2008; 336: 601-605.

30. Meissner K, Distel H, Mitzdorf U. Evidence for placebo effects on physical but not on biochemical outcome parameters: a review of clinical trials. BMC Med 2007; 5: 3.

\section{*Correspondence to}

Yurong Wang

College of Traditional Chinese Medicine

Jinan University

601 Huangpu West Avenue

Guangzhou

Guangdong

China

Ronghua Zhang

College of Traditional Chinese Medicine

Jinan University

601 Huangpu West Avenue

Guangzhou

Guangdong

China 